\title{
Piezoelectric Energy Harvesting Based on Bi-Stable Composite Laminate
}

\author{
Fuhong Dai and Diankun Pan \\ Additional information is available at the end of the chapter
}

http://dx.doi.org/10.5772/intechopen.76193

\begin{abstract}
Energy harvesting employing nonlinear systems offers considerable advantages comparing to linear systems in the field of broadband energy harvesting. Bi-stable piezoelectric energy harvesters have been proved to be a good candidate for broadband frequency harvesting due to their highly geometrically nonlinear response during vibrations. These bi-stable energy harvesters consist of bi-stable structure and piezoelectric transducers. The nonlinear response depends on the host bi-stable structure. A possible category of bistable structures is bi-stable composite laminate, which has two stable equilibrium states resulting from the mismatch in thermal expansion coefficients between plies. It has received considerable interest in deformable structure since the "snap-through" between two stable states results in a significant deformation without continuous energy supply. Combining piezoelectric transducer and the bi-stable composite laminate is a feasible method to obtain bi-stable energy harvester. Piezoelectric energy harvesters based on bistable composite laminate have been shown to exhibit high levels of power output over a wide range of frequencies. This chapter aims to summarize and review the various approaches in piezoelectric energy harvesting based on bi-stable composite laminates.
\end{abstract}

Keywords: energy harvesting, bi-stable, piezoelectric, laminate, nonlinear

\section{Introduction}

With the advent of low power, wireless and autonomous sensors, energy harvesting which is considered as a potential way to replace batteries and realize self-powering becomes a highly active research area [1,2]. Piezoelectric materials can be embedded in the host structure to convert the strain energy of the host structure into electrical energy through direct piezoelectric effect, so piezoelectric energy harvesting technique becomes one of the primary methods to 
harvest vibration energy. Compared with electromagnetic and electrostatic methods, the main advantages of piezoelectric energy harvesting technique are the larger power density and higher flexibility of being integrated into one system [3]. Because of the simple structure and ease of producing relatively high average strain for a given force input, the typical piezoelectric energy harvester consisting of a cantilever beam with piezoelectric elements attached near its clamped end is widely analyzed and designed. The cantilever type harvester operates on the fundamental principle of linear resonance. It means that the maximum energy transduction from the vibration source to harvester can be achieved by tuning the host beam's natural frequencies to be equal or very close to the excitation frequency. Therefore, the frequency bandwidth of linear harvester is usually limited to a specific range and the power output of linear harvesters would be reduced drastically when the frequency of vibration source deviates slightly from the resonant frequency of the harvesters. However, the energy of ambient vibrations is distributed over a broad spectrum of frequencies or the dominant frequencies drift with time in many applications. Several solutions were presented to solve this problem. Tuning mechanism is a potential method for broadband energy harvesting, which uses passive or active means to vary the fundamental frequency of the harvester to match the dominant frequency of the vibration source $[4,5]$. However, it is not very efficient when the frequency of vibration is random or varies rapidly, and tuning mechanism requires external power or complicated design. Nonlinear harvesters have been proposed for broadband energy harvesting benefiting from the ability of nonlinearities to extend the coupling between the excitation and a harmonic oscillator to a broader range of frequency.

The nonlinear harvester with a bi-stable potential is proved to be a good candidate for broadband energy harvesting. It has been shown that when carefully designed, bi-stable energy harvesters can provide significant power levels over a wide range of frequencies under steady-state harmonic excitation. Bi-stable systems have two stable equilibrium positions between which they may snap-through under a certain level of excitation. Generally, the bistable harvester can be achieved by applying magnetic force [6] or axial load [7] to buckle the piezoelectric beam. The potential energy of bi-stability can be tuned by the magnitude of magnetic force or axial load. However, such a magnetic bi-stable system would require an obtrusive arrangement of external magnets and could generate unwanted electromagnetic fields [8]. An alternative bi-stable composite laminate has been developed for broadband energy harvesting. This review aims to review the piezoelectric energy harvesting technologies based on two different bi-stable composite laminates and find out the potential benefits and defects from the existing energy harvesting techniques using bi-stable composite laminates.

\section{Bi-stable composite laminate}

The bi-stable asymmetric composite laminate was reported by Hyer firstly in 1981 [9, 10]. He found that thin asymmetric laminate may have two stable cylindrical shapes, which are attributed to the thermal stresses due to the difference of thermal expansion of the laminate. Due to the limitation of the classical lamination theory which predicts that all asymmetric laminates have a curved saddled shape where the two curvatures are always of opposite sign, 
Hyer developed a theory to explain the characteristics of the curved shapes of thin asymmetric laminates. This theory introduced the von-Karman geometric nonlinearities within the classical lamination theory to capture room-temperature shapes, and Rayleigh-Ritz method based on the concept of minimum total potential energy was used to obtain the curved shapes. The prediction of room-temperature shapes of asymmetric laminate becomes one of major research directions in next decades. The analytical model can be improved by more reasonable hypothesis for displacements and strains, so accuracy and efficiency can be achieved in a good balance [11, 12]. Additionally, the development of finite element method makes it possible to predict room-temperature shapes of asymmetric laminate with more complex geometry conditions [13, 14]. Another interesting feature of bi-stable laminate is snap-through behavior, which was studied extensively $[15,16]$. Recently, the potential applications of the bi-stable laminate have received considerable attention. One of potential applications is morphing structure. The main advantage of the bi-stable laminate as a morphing structure is that it has two stable positions where the structure can maintain without demanding an external power. Moreover, it only needs a very small energy input to trigger it snap from one stable position to the other with a relatively large deflection. Many researchers have studied the feasibility of bistable laminate as morphing structure from different perspectives, such as actuation method [17] and dynamics [18]. Another potential application of the bi-stable laminate is energy harvesting. Bi-stable laminate can provide large structural deformation resulting from the snap-through behavior. The piezoelectric elements will obtain large strain and produce high electric power if the piezoelectric elements are attached to the surface of bi-stable laminate. Compared to using magnetic mechanism, this bi-stable energy harvester has four main advantages: (1) the arrangement can be designed to occupy a smaller space; (2) there are no magnetic fields; (3) the laminate can be easily combined with piezoelectric materials; (4) there is potential to control over harvester response through adjusting lay-up and geometry [19].

According to the lay-up and stable shapes, bi-stable harvester based on composite laminate can be classified into two categories. The first one is the asymmetric laminate-based harvester. This asymmetric bi-stable laminate is made from a carbon fiber reinforced polymer (CFRP) with a $[0 / 90]_{\mathrm{T}}$ layup, as shown in Figure 1(a). For asymmetric laminate, the residual thermal stress causing curved deformation results from the differences in the thermal expansion coefficient between the carbon fiber and epoxy matrix during cooling process from an elevated cure temperature to room temperature. When the ratio of edge length to thickness increases to

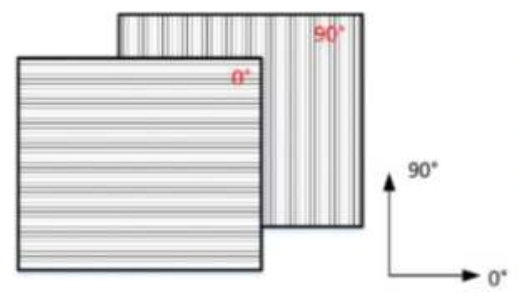

(a)
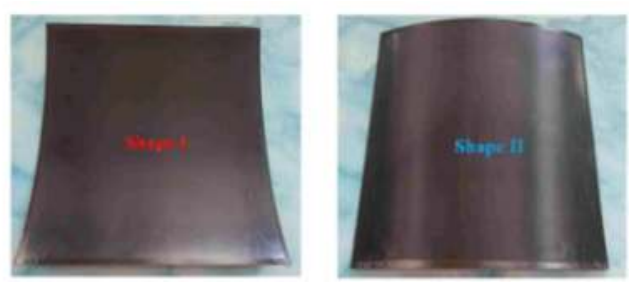

(b)

Figure 1. (a) Stacking sequence $[0 / 90]_{\mathrm{T}}$ of the asymmetric laminate; (b) two stable shapes of the asymmetric laminate. 


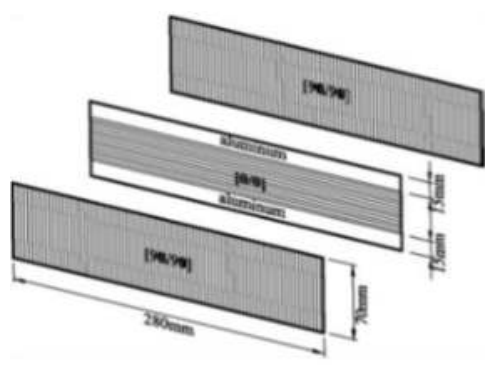

(a)

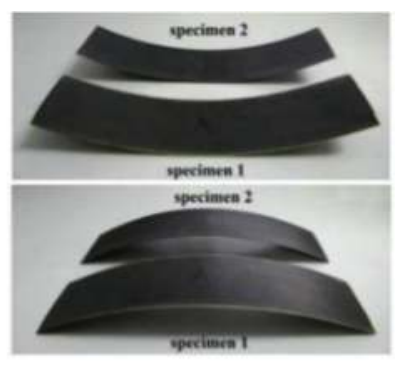

(b)

Figure 2. (a) Symmetric stacking sequence $\left[90_{2} / \mathrm{Al} / 90_{2}\right]_{\mathrm{T}} \cup\left[90_{2} / 0_{2} / 90_{2}\right]_{\mathrm{T}} \cup\left[90_{2} / \mathrm{Al} / 90_{2}\right]_{\mathrm{T}}$ for a bi-stable hybrid symmetric laminate; (b) stable shapes of two bi-stable hybrid symmetric laminate [20].

a specific value, asymmetric laminates has two approximately cylindrical stable shapes, as shown in Figure 1(b). The curvature direction of two shapes is orthogonal to each other, but the magnitude of curvature is equal to each other.

The other category is the hybrid symmetric laminate-based harvester. Li et al. [20] presented a bi-stable hybrid symmetric laminate (BHSL) by combining of aluminum plies and CFRP plies. The bi-stability is induced by the difference in thermal expansion coefficients between aluminum and CFRP. Typically, the stacking sequence of BHSL is shown in Figure 2(a). There are two types of region, which are the hybrid region with $\left[90_{2} / \mathrm{Al} / 90_{2}\right]_{\mathrm{T}}$ and composite region with $\left[90_{2} / 0_{2} / 90_{2}\right]_{\mathrm{T}}$. The stacking sequence of two regions is symmetric. This hybrid symmetric laminate exhibits two stable cylindrical shapes with same longitudinal curvature in the opposite direction, as shown in Figure 2(b).

\section{Piezoelectric energy harvesting based on bi-stable laminate}

\subsection{Asymmetric laminate-based harvester}

Asymmetric laminate has been investigated for several decades. The prediction of roomtemperature shape and bifurcation phenomena with different parameters (layup, geometry, thickness and so on) can be achieved a reasonable level of accuracy with reliable theoretical model and finite element method. The new challenge for asymmetric laminate is to find a suitable application scenario according to its particular features. The application of morphing structure was firstly presented, for example, morphing airfoil [21] and trailing edge box [22]. Though asymmetric laminate is a good candidate for morphing structure, how to trigger the snap-through behavior becomes a problem. To address this problem, many types of research have studied the snap-through motion of asymmetric laminate with different methods, such as piezoelectric actuation [23], memory alloy actuation [24] and heating actuation [25]. The method adopted in piezoelectric actuation is that applying voltage on piezoelectric patches attached to the surface of laminate enables piezoelectric patches to deform. Thus, asymmetric laminate can snap once the applying voltage is high enough. Piezoelectric actuation utilizes the 
direct piezoelectric effect. On the contrary, the voltage will be generated by piezoelectric patches resulting from inverse piezoelectric effect when asymmetric laminate deforms under external excitation. Therefore, the application of asymmetric laminate extends to piezoelectric energy harvesting.

Arrieta et al. [26] firstly presented a piezoelectric nonlinear broadband energy harvester based on asymmetric laminate in 2010. Only the experimental results were reported in this earlier work. This square $200 \times 200 \mathrm{~mm}$ asymmetric laminate with $\left[90_{2} / 0_{2}\right]_{\mathrm{T}}$ layup was mounted from its center to an electromechanical shaker, and four PZT-5A flexible piezoelectric patches were bonded to the surface of the laminate, as shown in Figure 3.

Five types of nonlinear responses were observed in the experiments. As shown in Figure 4(a), these five types of responses are linear oscillation, large amplitude limit cycle oscillation (LCO), chaotic oscillation, intermittency oscillation and 1/2 subharmonic oscillation. Large amplitude LCO, chaotic oscillation, and intermittency oscillation involve snap-through behavior between two stable shapes, so the voltage output corresponding to these three types of

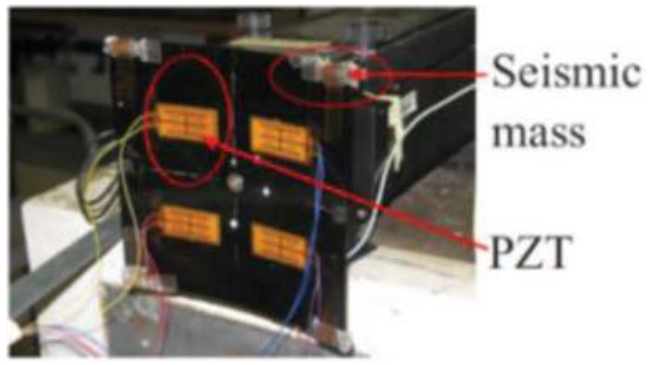

Figure 3. Piezoelectric energy harvester based on asymmetric laminate [26].

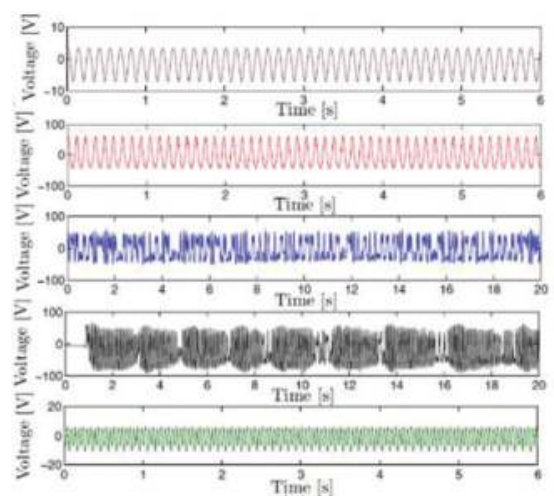

(a)

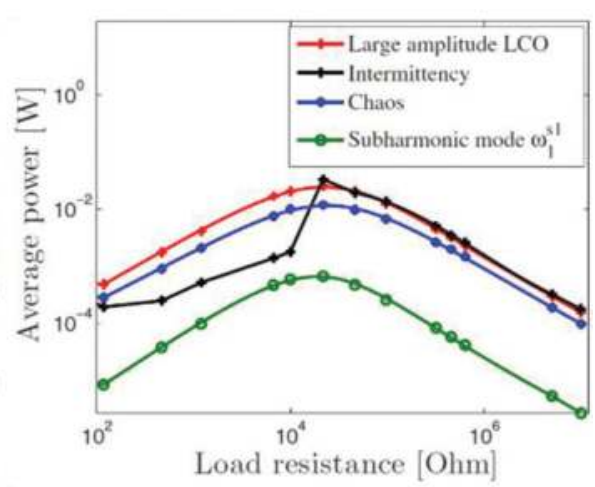

(b)

Figure 4. (a) Different responses for a forcing level $2 \mathrm{~g}$ : Linear response at $7.5 \mathrm{~Hz}$; large amplitude LCO at 8.6 Hz; chaotic oscillations at $12.5 \mathrm{~Hz}$; intermittency oscillations at $9.8 \mathrm{~Hz} ; 1 / 2$ subharmonic oscillations at $20.2 \mathrm{~Hz}$. (b) Experimental average power vs. load resistance for different responses [26]. 
responses are apparently higher than remaining two responses. Accordingly, the output powers of these three types are also higher, as shown in Figure 4(b). The intermittency and large amplitude LCO can give 34 and $27 \mathrm{~mW}$ respectively under a forcing acceleration level of $2.0 \mathrm{~g}$. For chaotic oscillation, the maximum average power was found to be $9 \mathrm{~mW}$. The experimental results in this work illustrate that asymmetric bi-stable laminate has rich dynamics for nonlinear energy harvesting and it is a potential candidate for broadband energy harvesting through combining the ranges where large amplitude LCO, chaotic oscillation, and intermittency oscillation occur.

Betts et al. [8, 27, 28] published several works to optimize configurations of asymmetric laminate for improving electrical power generation in 2012. This piezo-laminate was considered in the actuation arrangement, as shown in Figure 5. The actuation force applied on the center of the laminate surface while displacements of all four corners were constrained in the zdirection. The optimization was based on the static states of the system, so the electrical energy output depending on the snap-through between two stable shapes. The M8557-P1 MFC was employed as a piezoelectric material. Maximization of electrical energy generated in two sets of four piezoelectric layers was as optimizable objective under several constraint conditions, such as laminate surface area, limitation of piezoelectric strain and bistability. Four variables were adopted which are ply orientation, single ply thickness, aspect ratio and piezoelectric surface area. It was found that square cross-ply laminates $\left[0^{\mathrm{P}} / 0 / 90 / 90^{\mathrm{P}}\right]_{\mathrm{T}}$ offered the most extensive energy outputs while the laminate curvatures were maximized and aligned with the piezoelectric polarization axis.

The results of optimization are meaningful for the design of bi-stable energy harvester based on asymmetric laminate, but the optimization was carried out from a static perspective. The energy harvesting from vibration is a dynamic problem, so the optimization results are not very suitable for dynamic situations. Therefore, Betts et al. [29] had done some preliminary works about the dynamic transition between stable states as the piezoelectric laminate was exposed to an oscillating mechanical force in the same year. It was found that thicker laminates produced higher levels of energy when snap-through is fully induced. Furthermore, the dynamic loading causes a higher level of strains of piezoelectric transducers, which may cause its failure.

Next year, Arrieta et al. [30, 31] presented a new concept for broadband energy harvesting. They changed the boundary condition from center fixation to cantilever for asymmetric

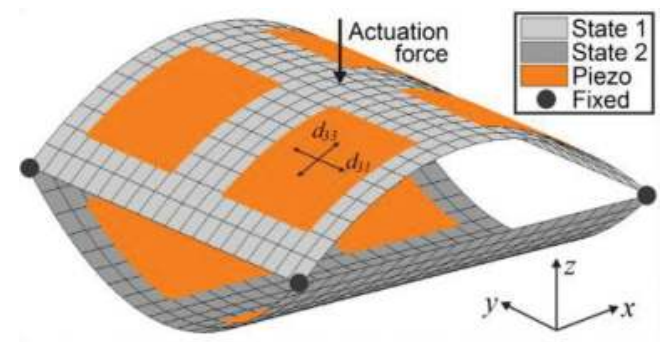

Figure 5. Actuation arrangement for a $\left[0^{\mathrm{P}} / 0 / 90 / 90 / 90^{\mathrm{P}}\right]_{\mathrm{T}}$ laminate with $40 \%$ piezoelectric coverage [27]. 
laminate. A symmetric-asymmetric layup was designed to realize cantilevered boundary condition, as shown in Figure 6(a). The symmetric layup was for clamping, and the asymmetric layup was for bi-stable laminate. Two flexible piezoelectric transducers (Piezo QP16n) were bonded on the surface of the asymmetric laminate and were closer to the clamped root. The two stable shapes of this cantilevered bi-stable laminate are shown in Figure 6(b). There are three significant advantages to this design. Firstly, this type of arrangement allows to exploit high strains developed close to clamped root by the piezoelectric transducers; Secondly, the cantilever configuration results in large displacements given the considerable distance from the root to the tip; Thirdly, the cantilevered configuration allows for more natural integration with the host structure.

Two specimens with different length were fabricated and tested respectively. The nonlinear behaviors of two specimens were studied by frequency sweep for a base acceleration, as shown in Figure 7 where blue dots and red crosses show frequency sweep with the initial condition on state 1 and 2 respectively, and arrows show regions of cross-well dynamics. Due to the cantilevered boundary condition, the two stable shapes are entirely asymmetric which leads to the different nonlinear responses depending on the initial state. Specimen B with longer length has a broader range of cross-well oscillation than specimen A. The cantilevered bi-stable laminate can be triggered to snap at a low level of base excitation. Additionally, the obtained power of this design ranges from 35 to $55 \mathrm{~mW}$ when Synchronized Switching Harvesting on Inductor (SSHI) circuit is adopted.

In the same year, Betts et al. [32] extended their work to experimental investigation of dynamic response and power generation characteristics of piezoelectric energy harvester based on a square $[0 / 90]_{\mathrm{T}}$ laminate with the size of $190 \times 190 \mathrm{~mm}$. A single piezoelectric Marco Fiber Composite (MFC) layer (M8585-P2, $85 \times 85 \mathrm{~mm}$ ) was attached to the laminate surface, as shown in Figure 8(a). Additional masses were attached to the four corners of the laminate to increase the achievable curvatures and help snap-through during oscillation. The whole device was mounted to the shaker from its center, as shown in Figure 8(b). The open-circuit voltage

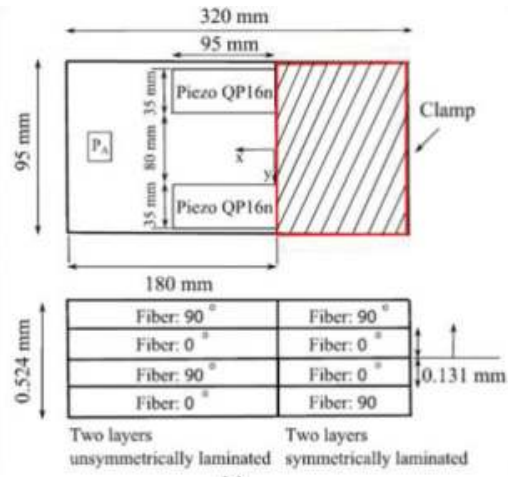

(a)

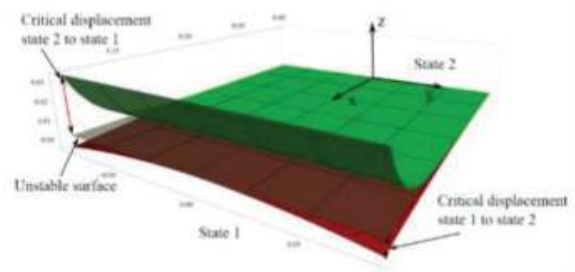

(b)

Figure 6. (a) Symmetric-asymmetric lay-up enabling cantilever configuration and positioning of piezoelectric transducers [30]. (b) Stable shapes and critical displacement for a cantilevered bi-stable laminate [31]. 


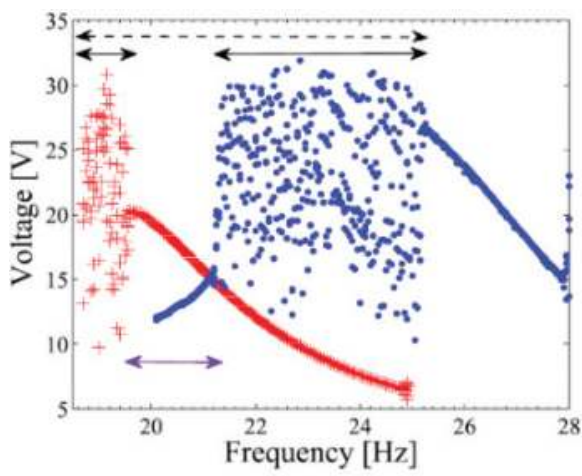

(a)

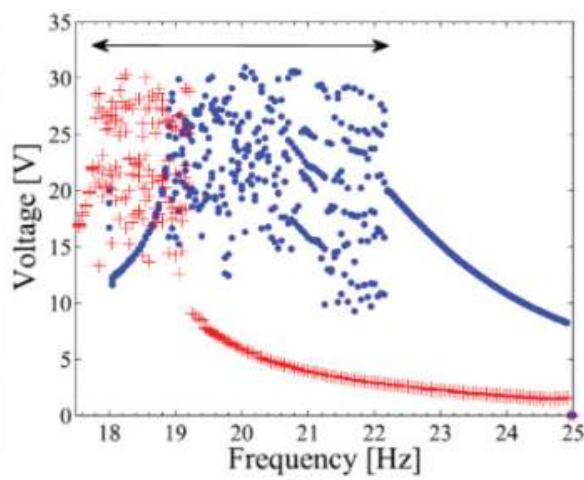

(b)

Figure 7. (a) Frequency-voltage response against forcing frequency of specimen $\mathrm{A}\left(L_{\mathrm{x}}=168 \mathrm{~mm}\right)$. Base acceleration level of $0.33 \mathrm{~g}$, and a load resistance of $66 \mathrm{k} \Omega$. (b) the frequency-voltage response against forcing frequency of specimen $\mathrm{B}$ $\left(L_{\mathrm{x}}=178 \mathrm{~mm}\right)$. Base acceleration level of $0.25 \mathrm{~g}$, and a load resistance of $66 \mathrm{k} \Omega$ [30].

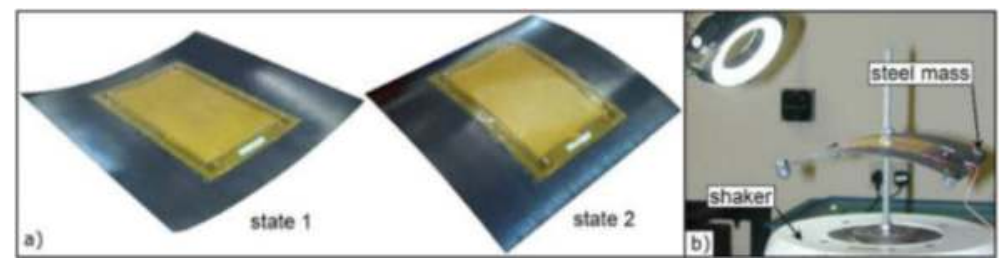

Figure 8. (a) Stable shapes of a $[0 / 90]_{\mathrm{T}}$ piezoelectric laminate; (b) experimental setup showing mechanical shaker attachment [32].

was measured, and the three-dimensional displacements were captured by a Digital Image Correlation (DIC) system. They had the similar experimental results as what was found by Arrieta et al. [26]. The experimental results have revealed that the modes of oscillation are sensitive to the frequency and amplitude of the external vibration, as shown in Figure 9. There are three oscillation patterns: (1) small-amplitude oscillation without snap-through; (2) uniform and nonuniform intermittent snap-through; (3) repeatable snap-through. The largest power output of $3.2 \mathrm{~mW}$ was found when snap-through occurred.

In 2014, Betts et al. [33] continued their work and presented an analytical model and experimental characterization of a piezoelectric bi-stable laminate of $200 \times 200 \times 0.5 \mathrm{~mm}$ with $[0 / 90]_{\mathrm{T}}$ stacking sequence. As before, a single flexible Macro Fiber Composite (MFC) was employed as a piezoelectric transducer and attached to one surface of the laminate. The analytical model was an extension of the model presented in Ref. [34]. This analytical model can capture the mix of nonlinear modes in the response of this bi-stable piezoelectric energy harvester subjected to mechanical vibrations. As before, these modes are continuous snap-through, intermittent snap-through (both periodic and chaotic), and small amplitude oscillations which were validated by experiments. 

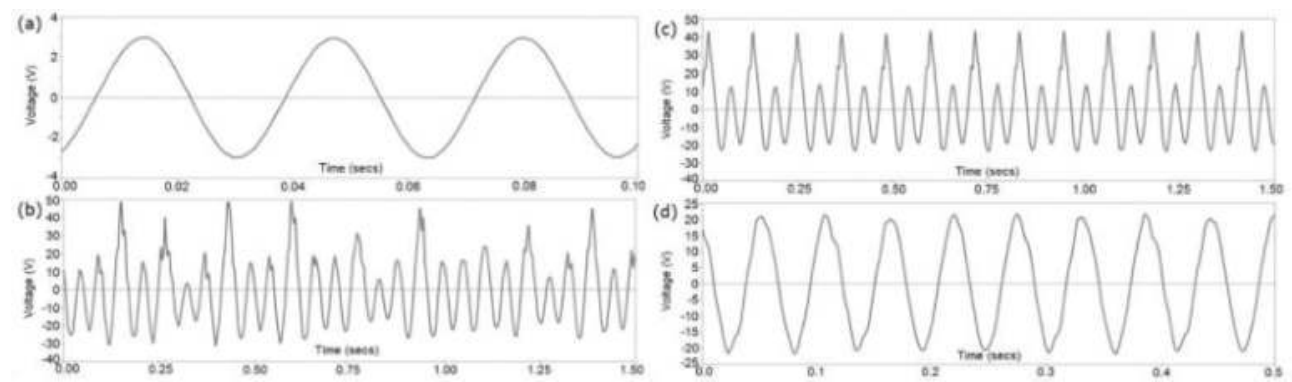

Figure 9. Different voltage outputs of bi-stable piezoelectric laminate: (a) low amplitude oscillations; (b) nonuniform behavior and chaotic snap-through; (c) intermittent snap-through; (d) repeated snap-through [32].

A map of these modes while varying the acceleration ( $\mathrm{g}$ level) and frequency of excitation was obtained based on experimental results, as shown in Figure 10(a). The results show that the desired continuous snap-through mode for energy harvesting requires high acceleration level, and intermittency snap-through modes can cover a broader range of frequency. The average powers with different frequencies and accelerations were measured and compared with analytical results. The analytical results are higher than experimental results, and analytical and experimental average powers for $10 \mathrm{~g}$ excitation are shown in Figure 10(b). This design can cover the bandwidth of $19.4 \mathrm{~Hz}$ involving snap-through behavior at the acceleration of $10 \mathrm{~g}$, and the peak power is as high as $244 \mathrm{~mW}$. Though the power output and bandwidth are so excellent, the acceleration level demanded is much higher than the work of Arrieta et al. [30].

In 2015, Syta et al. [35] employed the same design as Betts et al. [33]. The difference is that " $0-1$ test" was introduced for the experimental works to identify the chaotic dynamics

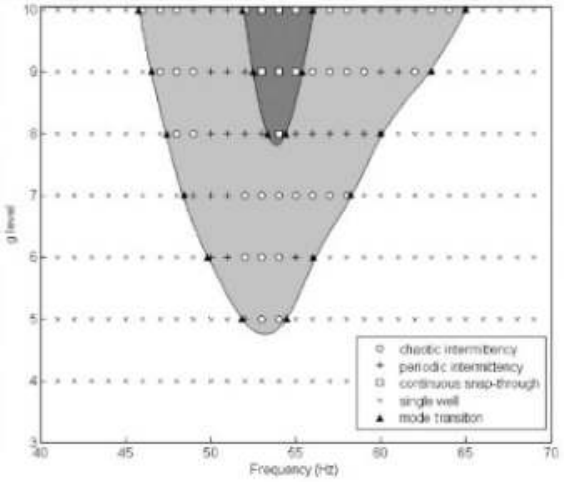

(a)

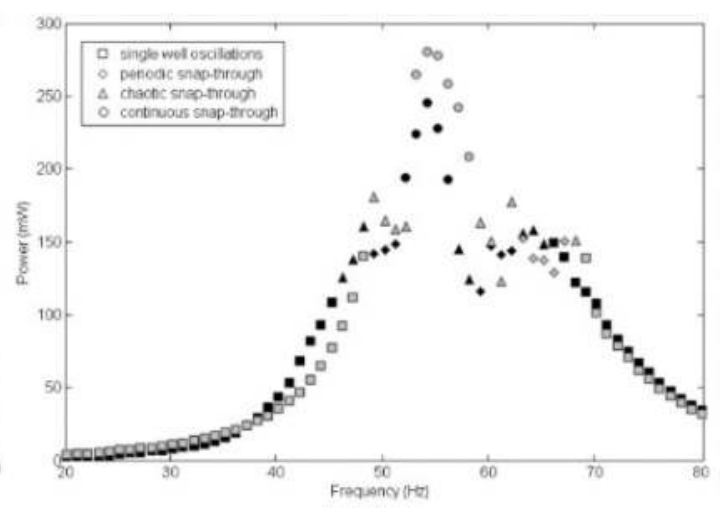

(b)

Figure 10. (a) Experimentally observed mode types associated with all combinations of drive frequency $(20-80 \mathrm{~Hz})$ and acceleration g-level (3-10 g); (b) average power outputs and associated modes for $10 \mathrm{~g}$ excitations. Experimental (black symbols) and modeling results (gray symbols) [33]. 
of this bi-stable energy harvester. Next year, Syta et al. [36] examined the modal responses of this bi-stable electro-mechanical energy harvester with same design by Fourier spectrum and Recurrence Quantification Analysis (RQA). RQA was used to identify the periodic and chaotic responses from reasonably short time series.

To overcome the high excitation level demand of large amplitude LCO at high frequencies, in 2015, Li et al. [37] exploited the nonlinear oscillations around the second vibration mode of a rectangular piezoelectric bi-stable laminate (RPBL) for broadband vibration energy harvesting at relatively higher frequencies with relatively lower excitation acceleration. This harvester consisted of a $150 \times 50 \times 0.42 \mathrm{~mm},\left[0_{2} / 90_{2}\right]_{\mathrm{T}}$ laminate and a $15 \times 15 \times 0.2 \mathrm{~mm}$ piece of PZT- $5 \mathrm{H}$ and copper electrode, as shown in Figure 11(a). Through finite element analysis, the frequency of second vibration mode of state A with mass is $66.8 \mathrm{~Hz}$, and the second vibration mode shape is shown in Figure 11(b). The experimental results show that the lowest excitation acceleration needed to trigger the LCO is $2.66 \mathrm{~g}$ at $59 \mathrm{~Hz}$. Two optimized RPBLs were found by finite element analysis, which illustrates that the frequency bandwidth of LCO can be broadened by decreasing the deformation needed to trigger the local snap-through. Although this design lowers the excitation, the power output of $0.98 \mathrm{~mW}$ is relatively low.

In 2014, Harris et al. [38] manufactured two piezoelectric energy harvesters and compared their performance by experiments. One of the two energy harvesters is linear with an asymmetric layup, as shown in Figure 12(a). The other one is bi-stable with asymmetric layup shown in Figure 12(b) (,) and the two stable states are shown in Figure 12. The results showed that the bi-stable harvester had higher power output over a broader range of frequencies at low frequency and low excitation and the linear one had the potential to produce a higher peak power but at a narrow bandwidth. In 2016, Harris et al. [19] continued their work and investigated the dynamics of this bi-stable energy harvester by multiscale entropy and " $0-1$ " test. As before, these oscillation modes including single-well oscillation, periodic and chaotic intermittent snap-through and continuous periodic snap-through were captured in experiments. The multiscale entropy and " $0-1$ " test can be helpful in the response characterization. One benefit from this analysis method is that the continuous plate system may be characterized by a single variable (voltage and displacement).

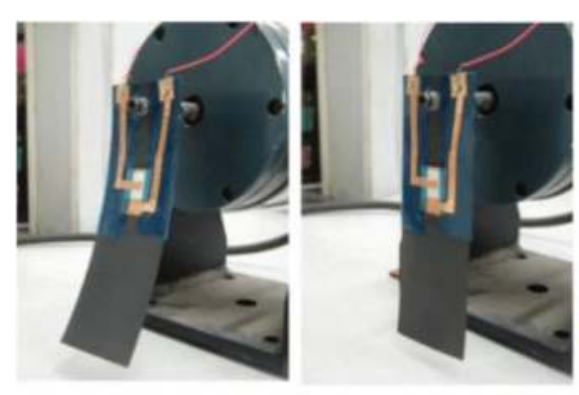

(a)

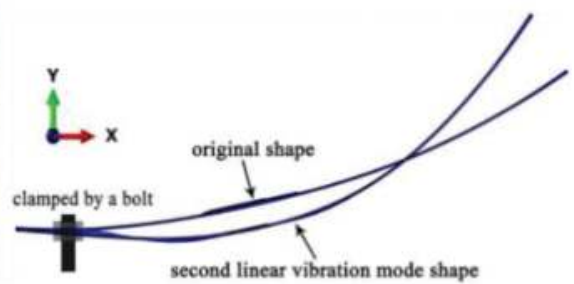

(b)

Figure 11. (a) Two stable states of RPBL; (b) the second vibration mode of stable state A (with proof mass) [37]. 


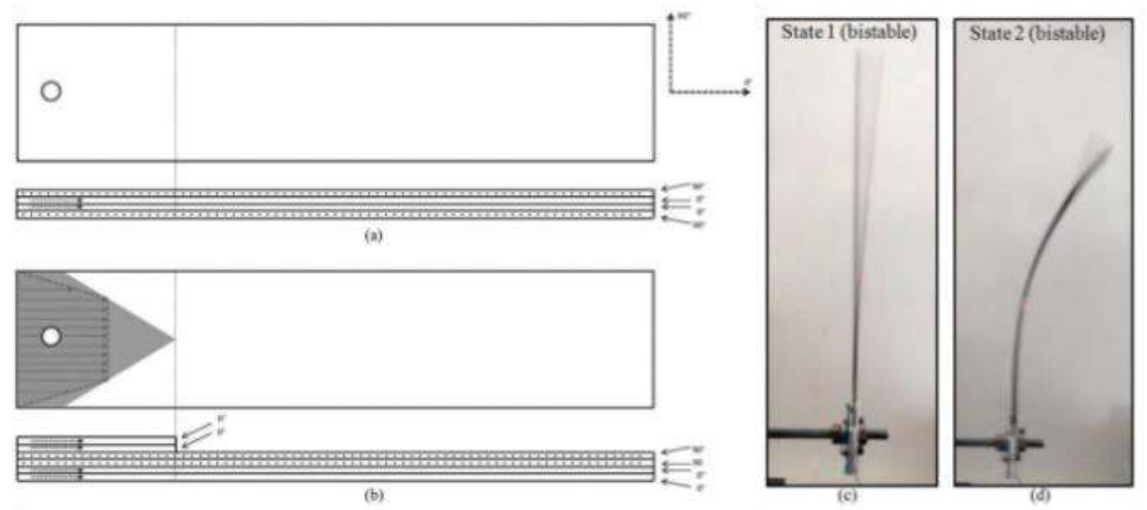

Figure 12. Laminate lay-ups: (a) linear and symmetric $[90 / 0 / 0 / 90]_{\mathrm{T}}$; and (b) bi-stable and asymmetric $[0 / 0 / 90 / 90]_{\mathrm{T}}$. The cantilevers were clamped at the left-hand side. Bi-stable states: (c) state I; and (d) state II [38].

In the same year, Harris et al. [47] added magnets in the bi-stable system to lower the level of excitation that triggers the snap-through for this bi-stable cantilever from one stable state to another, as shown in Figure 13. The system performance can be adjusted by varying the separation between the magnets. The scenario without magnets was taken as a control, and the different separations were measured. The results showed that this approach could adjust the fundamental frequency of the harvester and the magnets benefited the increase of the bandwidth with lower peak power at low acceleration levels and benefited the increase of peak power with narrower bandwidth at higher excitation levels. Additionally, a single-degree-of-freedom (SDOF) model was established based on the experimental load-deflection characteristic.

\subsection{Hybrid symmetric laminate-based harvester}

Besides traditional asymmetric bi-stable laminate, bi-stable laminates with novel layup supply new potential ways to design energy harvester. In 2015, Pan et al. [40] presented a bi-stable piezoelectric energy harvester (BPEH) based on bi-stable hybrid symmetric laminate (BHSL). The most apparent difference between asymmetric bi-stable laminate and BHSL is the stable shape. BHSL has two double-curved shapes, which have identical curvatures with opposite signs, as shown in Figure 2. It has better designability compared with the traditional

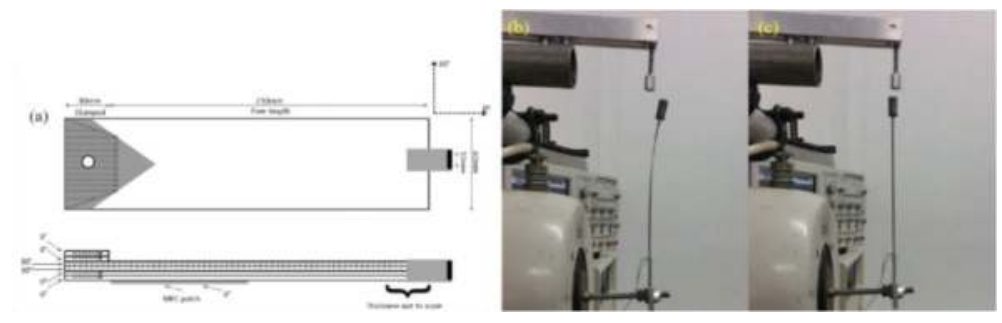

Figure 13. (a) Detail of the ply orientations, magnet location, and MFC location; (b) stable state I and (c) stable state II [39]. 
asymmetric laminate due to the variation of the position of the metallic layer. Moreover, the cantilever-type boundary condition can be realized benefiting from its unique symmetric stable shapes. In this work, to obtain more deformation, 20 pieces of PZT-5H were bonded to the middle of two BHSL's surfaces where the curvatures distribute uniformly. Due to the electrical conductivity of carbon fiber, the laminate can be as an electrode, and a parallel connection was employed for piezoelectric transducers. Two types of stacking sequences and two types of piezoelectric transducer shapes with the identical area were chosen, and four types of bi-stable harvesters and one linear harvester (LPEH) as control sample were designed, as shown in Figure 14. Through finite element analysis, it was found that stress mechanism of PZTs consists of bending stress and residual thermal stress and the stable shapes cannot reflect the deformation of the PZT. A rough power measurement, which is handshaking to actuate continuous snap-through behavior, was employed. The maximum power of $37.06 \mathrm{~mW}$ with the optimal resistance of $40 \mathrm{k} \Omega$ was obtained at $5 \mathrm{~Hz}$ from the $\mathrm{BPEH}$ with highest open-circuit voltage. Additionally, through the comparison between BPEHs and LPEH, it was shown that the BPEH could take advantages of each piece of PZT. It means the PZTs on BHSL can obtain uniform deformations during the snap-through process, which leads that BPEH can output much higher power.

Through previous preliminary investigation, BPEH shows good potential for energy harvester. In 2017, Pan et al. [41] continued their work and investigated the dynamics of this bi-stable energy harvester. The bi-stable laminate was redesigned with a smaller size of $100 \times 40 \mathrm{~mm}$ and 8 pieces of PZT-5H with $1.0 \times 1.0 \mathrm{~mm}$ were bonded in the middle of laminate surface like before. Three types of linear harvesters with two different layups and two types of PZT positions were designed as control samples, as shown in Figure 15.

The forward sweeps and reverse sweeps at five acceleration levels were carried out for $\mathrm{BPEH}$, as shown in Figure 16. Unlike responses of three linear harvesters, which only has have one sharp peak, responses of BPEH exhibited nonlinear characteristics. BPEH had a softening response under low level of excitation, and it could switch to hardening response when the excitation increased to a certain extent. Two oscillation modes were observed in experiments, which were single-well oscillation and cross-well oscillation. BPEH only has single-well oscillation mode under softening response at relatively low excitation level. When the response switch to hardening, BPEH can repeatedly travel between its two

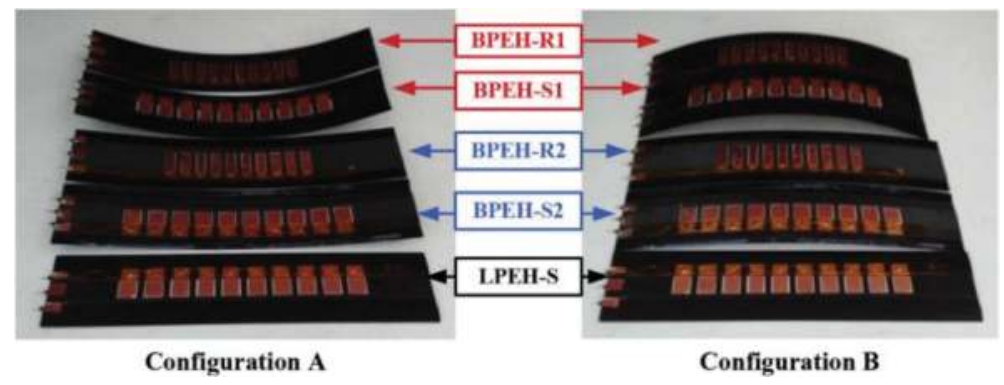

Figure 14. Stable shapes of the BPEHs and LEPH samples [40]. 


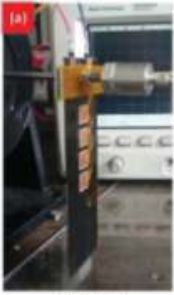

LPEH-1

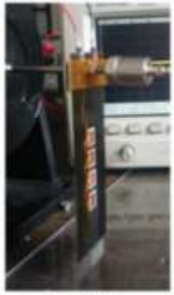

LPEH-2

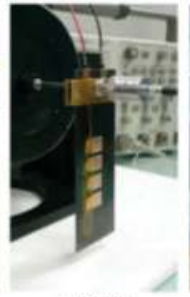

LPEH-3

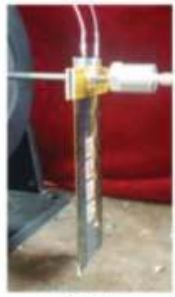

BPEH

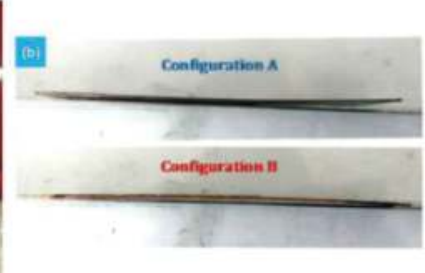

Figure 15. (a) Experimental setup showing mechanical shaker attachment of three types of LPEH and BPEH; (b) two stable configurations of BPEH [41].
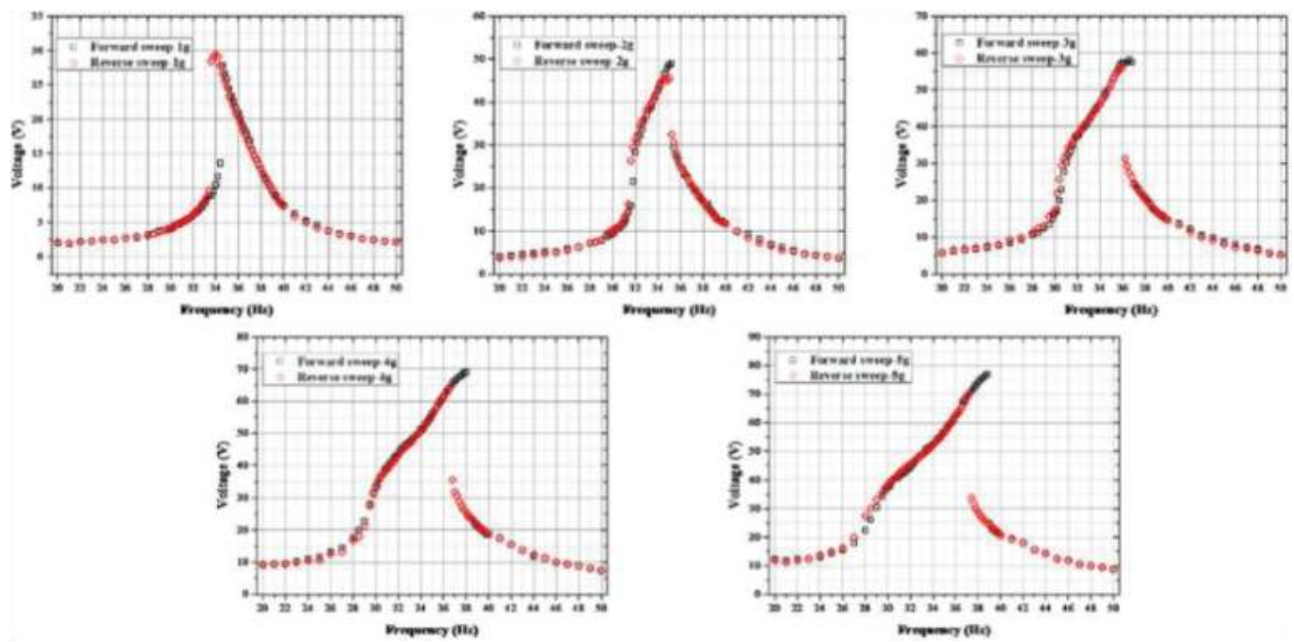

Figure 16. Experimental frequency-voltage responses of BPEH for acceleration ranging from 1 to $5 \mathrm{~g}$ [41]

potential wells, namely be under cross-well oscillation mode. When BPEH is under crosswell oscillation mode, the output voltage is much higher than that of single-well oscillation mode. The responses also are affected by sweep direction. The frequency range of highvoltage branch in the forward sweep is wider than that in the reverse sweep when BPEH exhibits hardening-type nonlinearity. Compared to LPEHs, BPEH has higher output voltage in a wider frequency range under the same excitation level. For output power, BPEH has a more remarkable performance than LPEHs. The maximum output power of BPEH subjected to $5 \mathrm{~g}$ acceleration at $36 \mathrm{~Hz}$ is 5.7 times more than that of LPEH-1. The reasons for this can be attributed to two aspects of higher voltage and more uniform strains in piezoelectric elements. The results of BPEH average power showed that the hardening responses help BPEH extend the high-output bandwidth and the output power associated with the excitation frequency and acceleration. All the experimental results demonstrated that BPEH has the potential to harvest vibration energy under broadband excitations. 


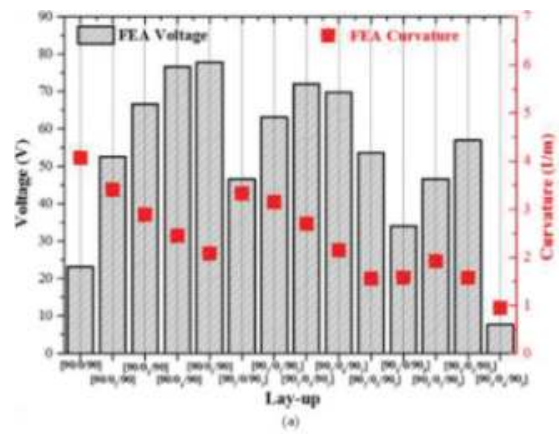

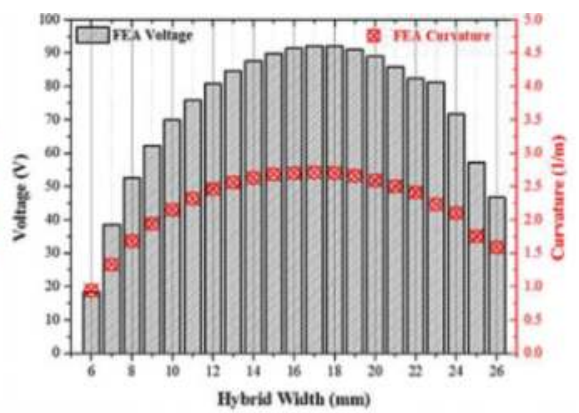

(b)

Figure 17. Initial voltages and longitudinal curvatures with (a) different lay-up and (b) hybrid width [42].

In the same year, Pan et al. [42] analyzed the influence of layup design on the performance of this bi-stable energy harvester. The initial voltage induced by stable configuration and longitudinal curvature with different lay-up and hybrid width was calculated and analyzed by a static finite element analysis. The results showed that the lay-up could vary the initial voltage and longitudinal in opposite directions, and hybrid width can adjust these two variables in the same direction, as shown in Figure 17. Through finite element analysis, it was found that the initial voltage of BPEH depends on strain variations in the two directions. Three types of $\mathrm{BPEHs}$ were manufactured to verify the analytical results. In the experiments, three types of oscillation modes were observed which are continuous cross-well vibration, single-well vibration, and intermittent cross-well vibration. The inherent characteristics of BPEH determine the frequency of characterized voltage, and the stable configuration affects the vibration mode. The BPEH with the lowest curvature can occur continuous snap-through vibration, but the $\mathrm{BPEH}$ with highest initial voltage only can occur single-well vibration. The layup and hybrid width can affect inherent characteristics and configurations at some time. The combination of lower frequency and lower longitudinal curvature is easier to obtain desired continuous crosswell vibration for energy harvesting.

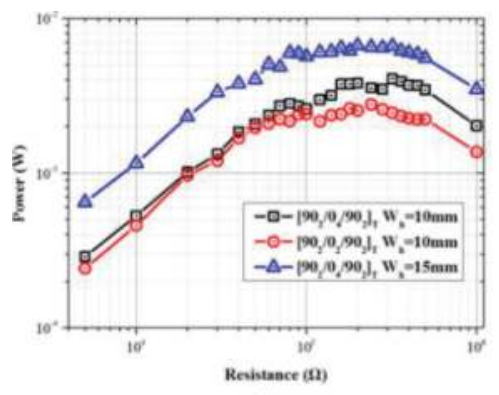

(a)

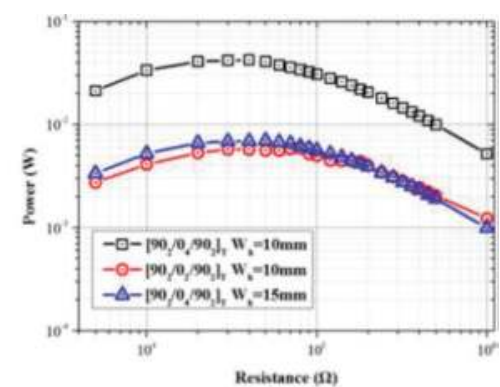

(b)

Figure 18. Experimental output power dependence of resistance for BPEHs (a) hand-driven method; (b) shaker-driven method [42]. 


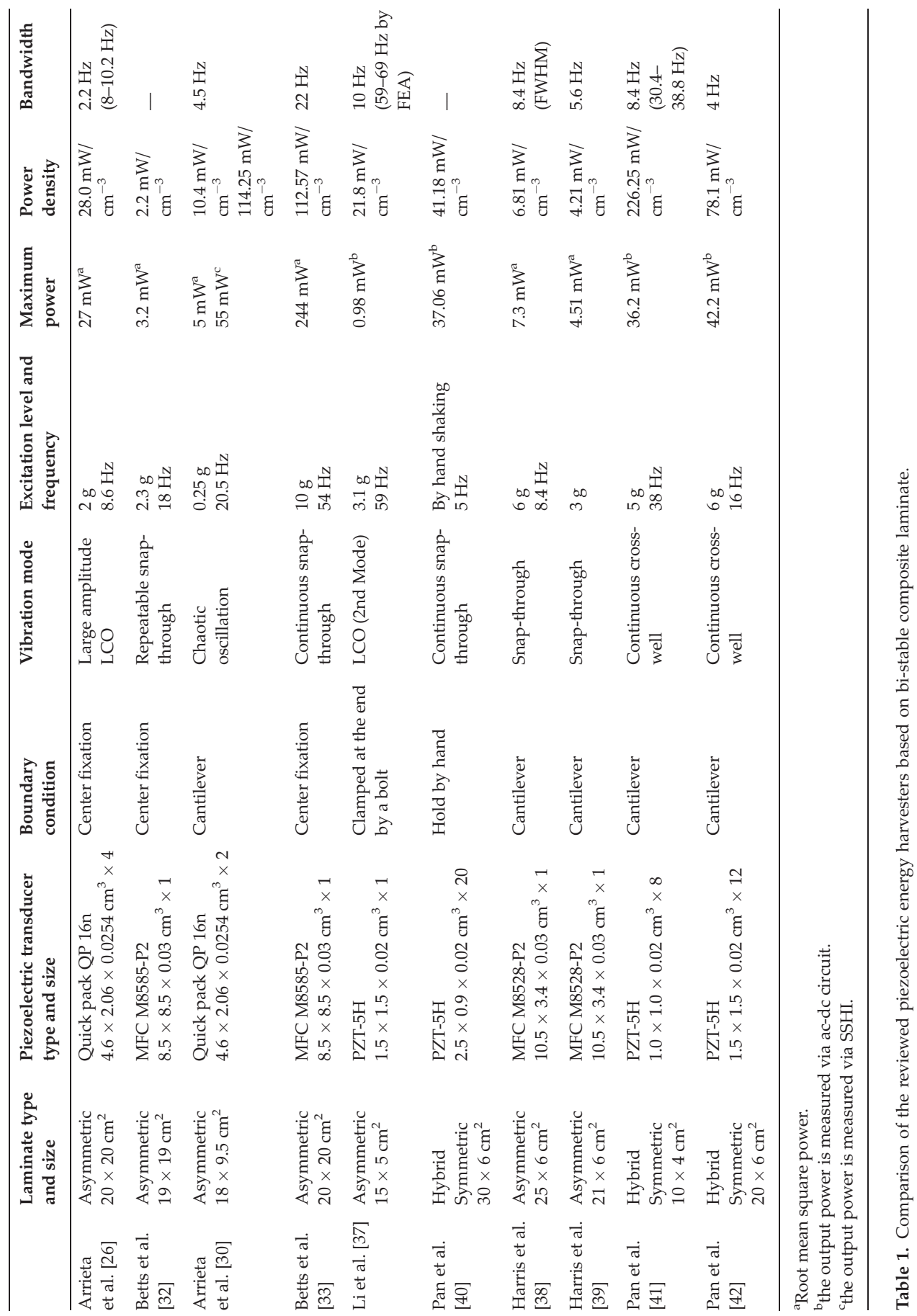


Three types of BPEH were actuated by two methods, which are a hand-driven method and a shaker-driven method, respectively. The hand-driven method was utilized to confirm the contribution of initial voltage to output power. As shown in Figure 18(a), BPEH with higher initial voltage can generate higher power by the hand-driven method as expected. However, the different results were found by the shaker-driven method, as shown in Figure 18(b). The $\mathrm{BPEH}$ under continuous cross-well vibration outputs the highest power. However, BPEH with the highest initial voltage only can occur single-well vibration so that it has the lower power. $\mathrm{BPEH}$, which is under intermittent cross-well vibration, generates the lowest maximum power.

\section{Summary}

This chapter reviewed the piezoelectric energy harvesting technique based on bi-stable composite laminate. As an essential branch of bi-stable energy harvesting, composite laminate has its unique advantages, such as inherent nonlinearity and combination with piezoelectric materials. The comparison of the reviewed piezoelectric energy harvester based on bi-stable composite laminate is shown in Table 1. Most of the designs are based on asymmetric laminate due to its more mature theory and design method. However, asymmetric laminate has observable bifurcation phenomena of size so that current designs have a relatively large size and it is hard to decrease the size. It may limit its further application. Hybrid laminate has a more flexible design and has an excellent potential to decrease the size. However, the theories including statics and dynamics of the hybrid laminate are still lacked. Also, most of the designs employ composite piezoelectric materials (MFC) as a transducer of which useful piezoelectric volume is limited. It is against to objective of decreasing the size and maintaining outputs. So far, the required excitation level of large amplitude oscillation (snap-through) is high. How to lower the required excitation with guaranteed reliability, stability and performance is a challenging task in both theory and practice. The harvesters based on bi-stable composite laminate have a relatively high output power (mW level) which can satisfy the requirement of conventional wireless sensors. It is difficult to evaluate their quality whether good or bad due to the different characterization method of power. However, portability design and low demanding are hot topics in this area of energy harvesting. Another way for harvester based on bi-stable laminate is finding suitable application situation, such as large amplitude and low-frequency vibration.

\section{Acknowledgements}

This work was supported by National Natural Science Foundation of China [grant No. 11372087], China Scholarship Council, Ph.D. Programs Foundation of Ministry of Education of China. 


\section{Author details}

Fuhong Dai* and Diankun Pan

*Address all correspondence to: daifh@hit.edu.cn

National Key Laboratory of Science and Technology on Advanced Composites in Special Environment, Harbin Institute of Technology, Harbin, China

\section{References}

[1] Kim HS, Kim J-H, Kim J. A review of piezoelectric energy harvesting based on vibration. International Journal of Precision Engineering and Manufacturing. 2011;12(6):1129-1141

[2] Sodano HA, Inman DJ, Park G. A review of power harvesting from vibration using piezoelectric materials. Shock and Vibration Digest. 2004;36(3):197-206

[3] Li H, Tian C, Deng ZD. Energy harvesting from low frequency applications using piezoelectric materials. Applied Physics Reviews. 2014;1(4):041301

[4] Roundy S, Zhang Y. Toward self-tuning adaptive vibration-based microgenerators. Smart Materials, Nano-, and Micro-Smart Systems, SPIE. 2005. pp. 373-384

[5] Challa VR, Prasad M, Shi Y, Fisher FT. A vibration energy harvesting device with bidirectional resonance frequency tunability. Smart Materials and Structures. 2008;17(1):015035

[6] Erturk A, Hoffmann J, Inman D. A piezomagnetoelastic structure for broadband vibration energy harvesting. Applied Physics Letters. 2009;94(25):254102

[7] Masana R, Daqaq MF. Relative performance of a vibratory energy harvester in mono-and bi-stable potentials. Journal of Sound and Vibration. 2011;330(24):6036-6052

[8] Betts DN, Kim HA, Bowen CR. Preliminary study of optimum piezoelectric cross-ply composites for energy harvesting. Smart Materials Research. 2012;2012:8. Article ID 621364. DOI: $10.1155 / 2012 / 621364$

[9] Hyer MW. Some observations on the cured shape of thin unsymmetric laminates. Journal of Composite Materials. 1981;15(2):175-194

[10] Hyer MW. The room-temperature shapes of four-layer unsymmetric cross-ply laminates. Journal of Composite Materials. 1982;16(4):318-340

[11] Cho M, Kim M-H, Choi HS, Chung CH, Ahn K-J, Eom YS. A study on the roomtemperature curvature shapes of unsymmetric laminates including slippage effects. Journal of Composite Materials. 1998;32(5):460-482 
[12] Jun W, Hong C. Cured shape of unsymmetric laminates with arbitrary lay-up angles. Journal of Reinforced Plastics and Composites. 1992;11(12):1352-1366

[13] Schlecht M, Schulte K, Hyer M. Advanced calculation of the room-temperature shapes of thin unsymmetric composite laminates. Composite Structures. 1995;32(1):627-633

[14] Schlecht M, Schulte K. Advanced calculation of the room-temperature shapes of unsymmetric laminates. Journal of Composite Materials. 1999;33(16):1472-1490

[15] M-L D, Hyer MW. The response of unsymmetric laminates to simple applied forces. Mechanics of Composite Materials and Structures: An International Journal. 1996;3(1):65-80

[16] Cantera M, Romera J, Adarraga I, Mujika F. Modelling and testing of the snap-through process of bi-stable cross-ply composites. Composite Structures. 2015;120:41-52

[17] Pirrera A, Avitabile D, Weaver P. On the thermally induced bistability of composite cylindrical shells for morphing structures. International Journal of Solids and Structures. 2012; 49(5):685-700

[18] Arrieta AF, Neild SA, Wagg DJ. On the cross-well dynamics of a bi-stable composite plate. Journal of Sound and Vibration. 2011;330(14):3424-3441

[19] Harris P, Bowen C, Kim A, Litak G. Dynamics of a vibrational energy harvester with a bistable beam: Voltage response identification by multiscale entropy and " $0-1$ " test. The European Physical Journal Plus. 2016;131(4):109

[20] Li H, Dai F, Weaver PM, Du S. Bi-stable hybrid symmetric laminates. Composite Structures. 2014;116:782-792

[21] Portela P, Camanho P, Weaver P, Bond I. Analysis of morphing, multi stable structures actuated by piezoelectric patches. Computers \& Structures. 2008;86(3):347-356

[22] Diaconu CG, Weaver PM, Mattioni F. Concepts for morphing airfoil sections using bistable laminated composite structures. Thin-Walled Structures. 2008;46(6):689-701

[23] Schultz MR, Hyer MW. Snap-through of unsymmetric cross-ply laminates using piezoceramic actuators. Journal of Intelligent Material Systems and Structures. 2003;14(12):795-814

[24] Dano ML, Hyer MW. SMA-induced snap-through of unsymmetric fiber-reinforced composite laminates. International Journal of Solids and Structures. 2003;40(22):5949-5972

[25] Li H, Dai F, Du S. Numerical and experimental study on morphing bi-stable composite laminates actuated by a heating method. Composites Science and Technology. 2012;72(14): $1767-1773$

[26] Arrieta A, Hagedorn P, Erturk A, Inman D. A piezoelectric bi-stable plate for nonlinear broadband energy harvesting. Applied Physics Letters. 2010;97(10):104102

[27] Betts DN, Kim HA, Bowen CR, Inman D. Optimal configurations of bi-stable piezocomposites for energy harvesting. Applied Physics Letters. 2012;100(11):114104 
[28] Betts DN, Kim HA, Bowen CR, Inman DJ. Optimization of piezoelectric bistable composite plates for broadband vibrational energy harvesting. Proc. SPIE 8341, Active and Passive Smart Structures and Integrated Systems. 2012, 83412Q (28 March 2012); DOI: 10.1117/12.930138; https://doi.org/10.1117/12.930138

[29] Betts DN, Kim HA, Bowen CR, Inman DJ. Static and dynamic analysis of bistable piezoelectric- composite plates for energy harvesting. In: 53rd AIAA/ASME/ASCE/AHS/ASC Structures, Structural Dynamics and Materials Conference, 2012-04-23 - 2012-04-26. American Institute of Aeronautics and Astronautics (AIAA). 2012

[30] Arrieta A, Delpero T, Bergamini A, Ermanni P. Broadband vibration energy harvesting based on cantilevered piezoelectric bi-stable composites. Applied Physics Letters. 2013; 102(17):173904

[31] Arrieta AF, Delpero T, Bergamini A, Ermanni P. A cantilevered piezoelectric bi-stable composite concept for broadband energy harvesting. Proc. SPIE 8688, Active and Passive Smart Structures and Integrated Systems 2013, 86880G (10 April 2013); DOI: 10.1117/ 12.2010122; https://doi.org/10.1117/12.2010122

[32] Betts DN, Bowen CR, Kim HA, Gathercole N, Clarke CT, Inman DJ. Nonlinear dynamics of a bi-stable piezoelectric-composite energy harvester for broadband application. The European Physical Journal Special Topics. 2013;222(7):1553-1562

[33] Betts DN, Bowen CR, Kim HA, Guyer RA, Le Bas P-Y, Inman DJ. Modelling the dynamic response of bi-stable composite plates for piezoelectric energy harvesting. 55th AIAA/ASME/ ASCE/AHS/ASC Structures, Structural Dynamics, and Materials Conference, 2014.2014-01-13 - 2014-01-17, National Harbor

[34] Diaconu CG, Weaver PM, Arrieta AF. Dynamic analysis of bi-stable composite plates. Journal of Sound and Vibration. 2009;322(4):987-1004

[35] Syta A, Bowen C, Kim H, Rysak A, Litak G. Experimental analysis of the dynamical response of energy harvesting devices based on bi-stable laminated plates. Meccanica. 2015;50(8):1961-1970

[36] Syta A, Bowen CR, Kim HA, Rysak A, Litak G. Responses of bi-stable piezoelectriccomposite energy harvester by means of recurrences. Mechanical Systems and Signal Processing. 2016;76-77:823-832

[37] Li H, Dai F, Du S. Broadband energy harvesting by exploiting nonlinear oscillations around the second vibration mode of a rectangular piezoelectric bi-stable laminate. Smart Materials and Structures. 2015;24(4):045024

[38] Harris P, Bowen CR, Kim HA. Manufacture and characterisation of piezoelectric broadband energy harvesters based on asymmetric bi-stable laminates. Journal of Multifunctional Composites. 2014;2(3):113-123

[39] Harris P, Litak G, Bowen CR, Arafa M. A composite beam with dual bistability for enhanced vibration energy harvesting. Proc. SPIE 9865, Energy Harvesting and Storage: 
Materials, Devices, and Applications VII, 98650K (17 May 2016); DOI: 10.1117/12.2225144; https://doi.org/10.1117/12.2225144

[40] Pan D, Dai F, Li H. Piezoelectric energy harvester based on bi-stable hybrid symmetric laminate. Composites Science and Technology. 2015;119:34-45

[41] Pan D, Ma B, Dai F. Experimental investigation of broadband energy harvesting of a bistable composite piezoelectric plate. Smart Materials and Structures. 2017;26(3):035045

[42] Pan D, Li Y, Dai F. The influence of lay-up design on the performance of bi-stable piezoelectric energy harvester. Composite Structures. 2017;161:227-236 